\title{
Hemoptysis and fever post-transplant: Diagnosis?
}

\author{
Marc G Romney MD
}

\begin{abstract}
A
28 year-old man was admitted to the hematology unit of a tertiary care hospital following an episode of hemoptysis and fever 170 days after an allogeneic bone marrow transplant. On admission, his temperature was $39.4^{\circ} \mathrm{C}$. The patient was thoroughly investigated for sepsis, and started on intravenous ciprofloxacin and ceftazidime. Total white blood count was $1.5 \times 10^{9} / \mathrm{L}$, with hemoglobin $85 \mathrm{mg} / \mathrm{L}$ and platelets $14 \times 10^{9} / \mathrm{L}$. A chest $\mathrm{x}$-ray was performed and provisionally reported as normal. During the months before admission, the patient's transplant was complicated by graft-versus-host disease and renal impairment. A recent episode of abdominal
\end{abstract}

pain was diagnosed as cytomegalovirus enteritis, for which he received intravenous ganciclovir $450 \mathrm{mg}$ daily for 14 days.

On review of the patient's chest $x$-ray, a faint nodule in the right upper lobe was discerned, which in retrospect had been present for two weeks. A computed tomographic scan of the patient's chest was performed, showing the lesion to be more obvious (Figure 1). Amphotericin B was started for a presumed invasive fungal infection.

After $72 \mathrm{~h}$ incubation, a blood culture from the original septic work-up yielded growth of a bacterial species.

What is the diagnosis?

Continued on page 316


Figure 1) Computed tomography scan of the chest, showing a cavitary lesion in the lung on the right side

Department of Medical Microbiology, Faculty of Medicine, University of British Columbia, Vancouver, British Columbia Correspondence: Dr Marc G Romney, Division of Medical Microbiology, St Paul's Hospital, 1081 Burrard Street, Vancouver, British Columbia V6Z1Y6. Telephone 604-872-9743,fax604-806-8661,e-mailmarcromney@hotmail.com 
Continued from page 312

\section{DIAGNOSIS}

The blood culture from the original septic work-up grew a branching, filamentous, Gram-positive rod. Over the following week, the same branching Gram-positive rod was recovered in three of six blood culture bottles (all aerobic). A modified Kinyoun stain was performed, and the organism was determined to be partially acid-fast. The organism was further identified as Nocardia asteroides.

While in hospital, the patient was started on high dose intravenous trimethoprim/sulphamethoxazole, and his condition gradually improved. He was discharged following three weeks of parenteral antimicrobial therapy, and then continued on oral maintenance antibiotics while at home. Despite the regression of the pulmonary nodule and sterilization of his blood, the patient continued to require periodic admissions to the hospital for numerous other complications from his bone marrow transplant.

\section{DISCUSSION}

Whenever branching, beaded, filamentous Gram-positive rods are cultured under aerobic conditions, the possibility of infection with Nocardia species should be raised. If the organisms are partially acid-fast, as was determined in this case, a presumptive identification of Nocardia species can be made. Growth can be observed on most simple media for bacteria, fungi and mycobacteria, while added carbon dioxide promotes more rapid growth. Nocardiae require a minimum of 48 to $72 \mathrm{~h}$ incubation before colonies become visible on solid media, and therefore culture plates should be incubated for a minimum of two weeks if a nocardial infection is suspected. Colonial morphology is extremely variable; most colonies have an earthy odour. Laboratories at larger institutions and reference laboratories usually identify Nocardia to the species level using conventional biochemical tests. The most common method (Gordon method) involves hydrolysis of casein, tyrosine, xanthine and hypoxanthine. Newer methods for direct identification of Nocardia species include ELISA techniques, recombinant DNA probes, and polymerase chain reaction-restriction fragment length polymorphism analyses. These methods remain investigational, and are not available for routine use.

The first documented case of nocardiosis (originally known as 'bovine farcy') was discovered in cattle by the French veterinarian Edmond Nocard in 1888 on the island of Guadeloupe. Soon thereafter, the first human infection in a patient with pneumonia and brain abscess was reported, and the organism was classified as $N$ asteroides. Although the classification of norcardiaceae is still evolving, $N$ asteroides is no longer considered a single species and can be divided into at least three subgroups: $N$ asteroides sensu stricto, Nocardia nova, and Nocardia farcinica. Other pathogenic Nocardia species include Nocardia brasiliensis, Nocardia transvalensis and Nocardia otitidiscaviarum.

As with this case, nocardiosis is primarily recognized in the immunocompromised host. With cell-mediated immunity playing a major role in defending the body against nocardial infection, the majority of infections are reported in patients who have received bone marrow or solid organ transplants, in patients with HIV/AIDS, or in patients with hematopoietic malignancies.

Based primarily on clinical experience, sulphonamides have become the mainstay of therapy in treating nocardiosis. The use of sulphonamides, however, is often complicated by adverse reactions, especially in the HIV/AIDS population. Imipenem/cilastatin, amikacin and other agents (based mainly on the results of in vitro susceptibility tests) are now being used more extensively to treat invasive nocardial disease.

\section{REFERENCES}

1. Lerner PL. Nocardiosis. Clin Infect Dis 1996;22:891-905.

2. McNeil MM, Brown JM. The medically important aerobic

Actinomycetes: epidemiology and microbiology. Clin Micro Rev 1994; 7:357-417. 


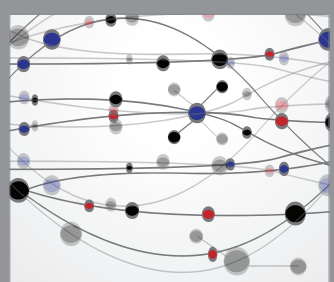

The Scientific World Journal
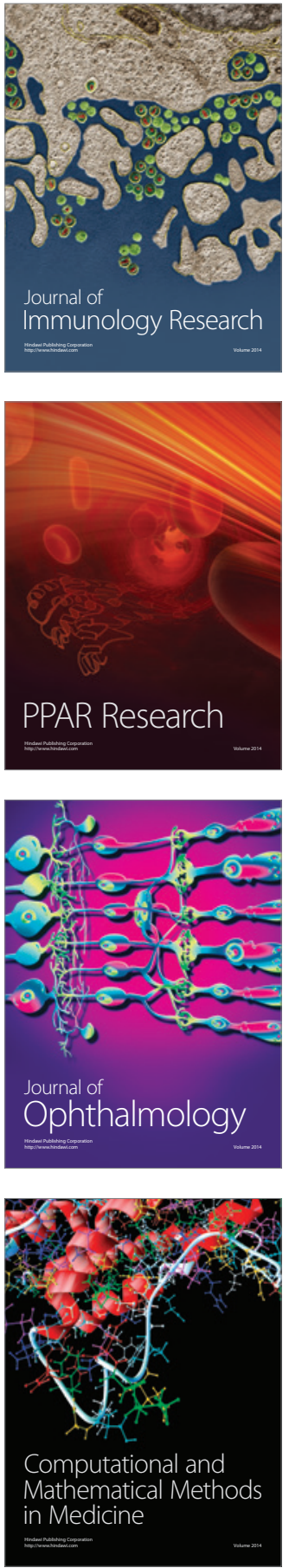



Gastroenterology Research and Practice

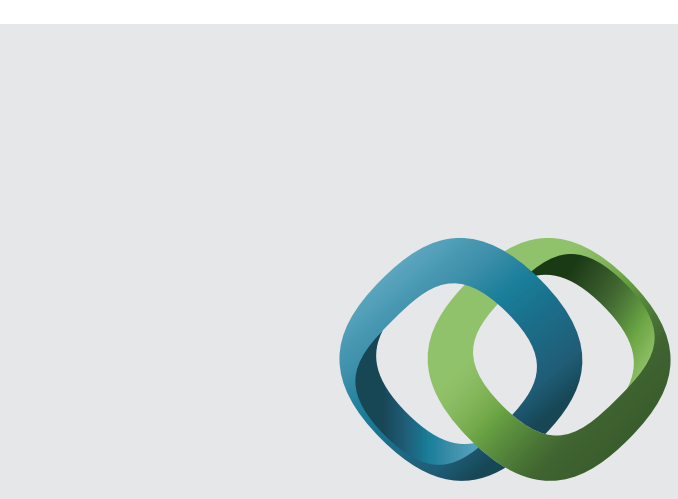

\section{Hindawi}

Submit your manuscripts at

http://www.hindawi.com
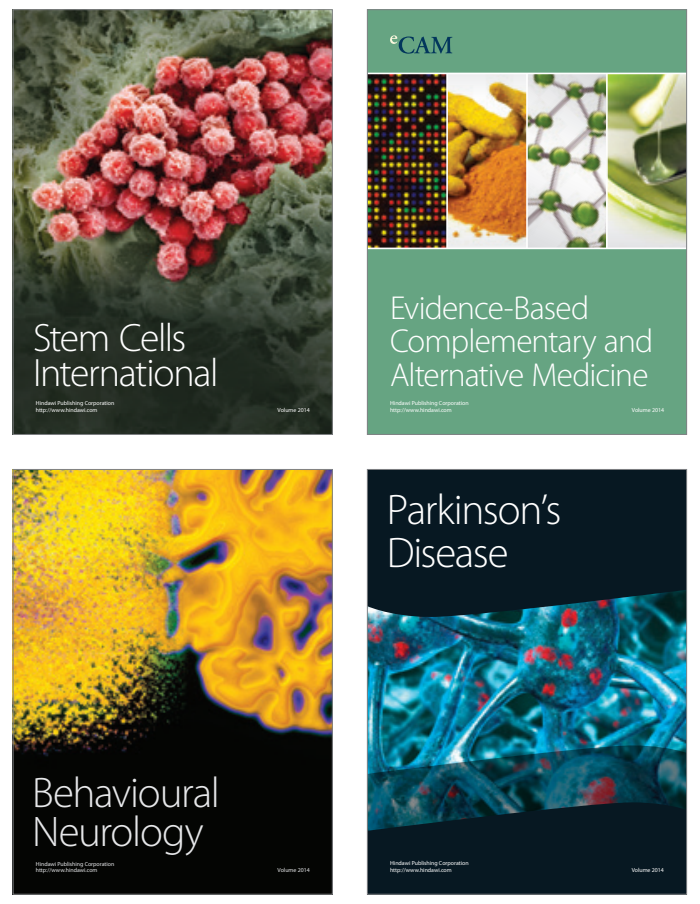
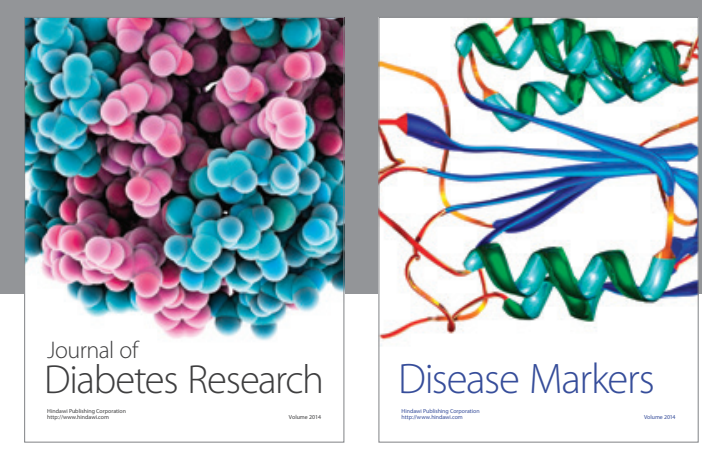

Disease Markers
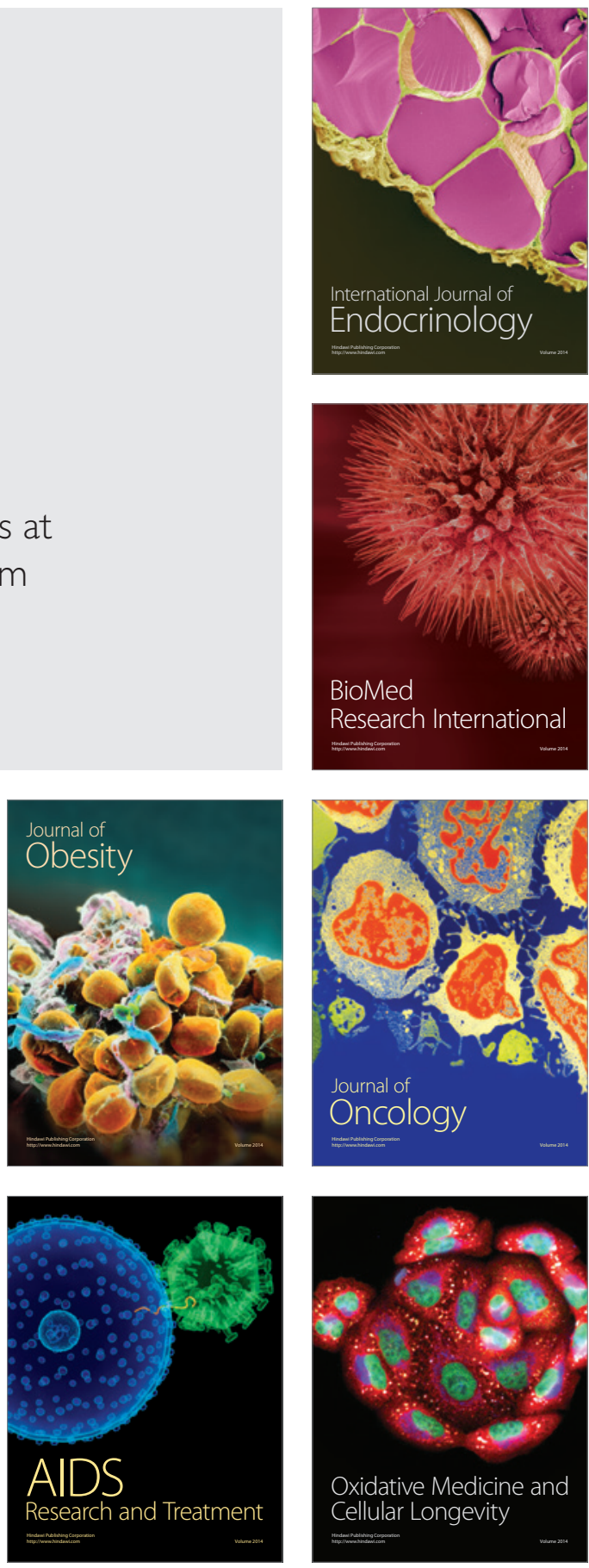